\title{
Gadolinium and systemic fibrosis: guilt by association
}

Jonathan Kay, ${ }^{1}$ László Czirják²

Nephrogenic systemic fibrosis (NSF, originally called 'nephrogenic fibrosing dermopathy') is a painful and debilitating fibrosing disorder that was first identified in 1997 in several patients with stage 5 chronic kidney disease (glomerular filtration rate $($ GFR $)<15 \mathrm{ml} / \mathrm{min} / 1.73 \mathrm{~m}^{2}$ or permanently requiring dialysis) who had undergone renal transplantation at Sharp Memorial Hospital in San Diego, California. ${ }^{1}$ It typically manifests with skin tightening, tethering and hyperpigmentation on the trunk and on the extremities, progressing from distal to proximal, and often results in fixed flexion contractures of the fingers, elbows, knees and ankles. More recently, extensive systemic involvement has been identified including fibrosis of lymph nodes, thyroid, oesophagus, heart, lungs, liver, diaphragm, skeletal muscle, genitourinary tract and dura mater. ${ }^{23}$ The presence of skin changes of NSF has been associated with a nearly threefold increased risk of death within 24 months. ${ }^{4}$

On skin biopsy, the dermis is hypercellular with increased numbers of dermal fibroblasts. ${ }^{5} 6$ Thickened collagen bundles aligned with intact elastic fibres extend from the superficial dermis through the subcutis along interlobular septa. ${ }^{6}$ Interstitial mucin deposition is increased. CD34 fibrocytes, presumably originating from the circulation, and occasional cells of monocytemacrophage lineage that stain for factor XIIIa and CD68 may be present in the dermis. When deeper tissue is sampled, fibrosis of subcutaneous tissue and fascia can also be observed. ${ }^{7}$ However, unlike scleroderma or scleromyxoedema, there is little or no inflammatory infiltrate in the skin of patients with NSF. Although the skin changes and joint contractures of NSF may resemble those of chronic

\footnotetext{
1Rheumatology Division, Department of Medicine, University of Massachusetts School of Medicine, Worcester, Massachusetts, USA

2Department of Immunology and Rheumatology, University of Pécs, Pécs, Hungary
}

Correspondence to Jonathan Kay, Rheumatology Center, UMass Memorial Medical Center, 119 Belmont Street, Worcester, MA 01605, USA; jonathan.kay@ umassmemorial.org graft-versus-host disease, NSF occurs in the absence of recent allogeneic haematopoietic cell transplantation; on skin biopsy, increased interstitial mucin and CD34 fibrocytes in the dermis typically differentiate NSF from chronic graftversus-host disease. $^{8}$

In 2006, Grobner reported that five of nine patients receiving haemodialysis in his unit developed NSF within days to weeks after undergoing MRI with Omniscan, a gadolinium-containing contrast agent. ${ }^{9}$ At that time, because of the lower risk of nephrotoxicity, gadolinium-containing contrast agents were assumed to be safer than iodinated contrast agents for administration to patients with chronic kidney disease. ${ }^{10}$ Thus, gadolinium-enhanced MR angiography of the lower extremities was often performed in patients with stage 5 chronic kidney disease to evaluate the condition of the peripheral arteries prior to renal transplantation. ${ }^{11}$ Gadoliniumcontaining contrast agents are composed of the rare earth metal gadolinium noncovalently bound to an organic chelating agent, with excess chelate added to bind free gadolinium that might have dissociated from the gadolinium-chelate complex. Omniscan is an aqueous solution that contains gadodiamide and excess free chelate caldiamide sodium. Magnevist is an aqueous solution that contains gadopentetate dimeglumine and excess free chelate diethylenetriamine pentaacetate. Grobner hypothesised that metabolic acidosis might destabilise the gadolinium-chelate complex, resulting in dissociation of gadolinium from chelate and deposition of gadolinium in tissue.

Following Grobner's insightful suggestion that NSF might be a complication of gadolinium-containing contrast agent administration in patients with stage 5 chronic kidney disease, several lines of investigation have established a strong causal relationship between exposure to gadolinium-containing contrast agents used in imaging procedures and the subsequent development of NSF. The prevalence of NSF among patients with stage 5 chronic kidney disease who had been exposed to a gadolinium-containing contrast agent has been estimated to be as high as $18 \%{ }^{12}$ to $30 \% .^{4}$ In a prospective case-control cohort study, Todd and colleagues observed skin changes characteristic of NSF in 13\% of 186 patients with stage 5 chronic kidney disease who were receiving haemodialysis in community-based centres. Among these patients, prior exposure to a gadoliniumcontaining contrast agent was strongly associated with the subsequent development of NSF skin changes (OR 14.7). ${ }^{4}$ The risk of developing NSF was both time- and dose-dependent in a different cohort of 36 patients with stage 5 chronic kidney disease and biopsy-proven NSF who had been exposed to Magnevist: NSF developed within 3 months after the last dose in $66 \%$ of patients and the likelihood was greater with higher cumulative and total doses of the gadolinium-containing contrast agent (OR 1.2). ${ }^{13}$ Gadolinium has been detected and quantified in biopsies of skin ${ }^{14}$ and other tissues ${ }^{3}$ from patients with NSF and in the skin of rats, which previously had been subjected to $5 / 6$ nephrectomy, after the intravenous administration of a gadolinium-containing contrast agent. ${ }^{15}$ Thus, the association between administration of gadolinium-containing contrast agents to individuals with chronic kidney disease and the subsequent development of NSF satisfies most of the Bradford-Hill criteria for causality. ${ }^{16}$ Demonstration of a plausible mechanism by which a gadolinium-containing contrast agent could induce the pathological changes of NSF would further substantiate this cause and effect relationship.

A clue to the pathophysiological mechanism by which tissue fibrosis develops after administration of a gadoliniumcontaining contrast agent is derived from the observation that skin thickening and tethering improve dramatically when patients with NSF are treated with imatinib mesylate. ${ }^{17}$ This small molecule tyrosine kinase inhibitor blocks signalling through both the transforming growth factor $\beta$ (TGF $\beta$ ) receptor and the platelet-derived growth factor (PDGF) receptor in normal and scleroderma dermal fibroblasts in vitro, thereby decreasing transcription and translation of type I collagen and fibronectin genes. ${ }^{18}$ In a murine model of bleomycin-induced dermal fibrosis, pretreatment with imatinib mesylate reduces extracellular matrix protein synthesis and dermal thickness ${ }^{18}$ and treatment with imatinib mesylate, beginning 3 weeks after the 
first intracutaneous bleomycin injection, not only stops further progression but also induces regression of pre-existing dermal fibrosis. ${ }^{19}$ TGF $\beta$ expression is increased in affected skin and muscle from patients with NSF compared with healthy controls. ${ }^{7}$ Incubation of normal human peripheral blood monocytes with either Omniscan, gadopentetate dimeglumine or gadolinium chloride stimulates the expression of a number of cytokines and growth factors, including TGF $\beta .^{20}$ Thus, exposure of patients with chronic kidney disease to gadoliniumcontaining contrast agents may induce the production of TGF $\beta$ and possibly of PDGF.

In this issue of Annals of the Rheumatic Diseases, two original reports from the same group describe additional effects of gadolinium-containing contrast agents on fibroblast and macrophage function that further contribute to understanding how a rare earth metal chelated by an organic ligand may induce tissue fibrosis. Piera-Velazquez and colleagues report direct effects of Omniscan and gadopentetate dimeglumine on dermal fibroblasts that are relevant to the pathogenesis of NSF. ${ }^{21}$ They found a prominent increase in type I collagen production after culture of normal dermal fibroblasts for $48 \mathrm{~h}$ with either gadolinium-containing contrast agent but not with the free chelate caldiamide sodium. They also showed markedly increased production of the extracellular matrix components types I and III collagen, fibronectin and hyaluronic acid by dermal fibroblasts cultured from patients with NSF compared with those from healthy controls. In three patients with NSF, fibroblasts cultured from clinically affected skin displayed an activated myofibroblast phenotype and produced greater amounts of COL1A1 mRNA and type I procollagen and collagen proteins than did fibroblasts cultured from clinically unaffected skin. After serial passages of these NSF dermal fibroblasts in culture, an 'activated' phenotype with increased expression of genes encoding extracellular matrix macromolecules was maintained. Taken together, these data suggest that persistent activation of dermal fibroblasts, perhaps resulting from a direct action of free gadolinium or of the gadolinium-chelate complex on the fibroblast, may account for the fibrosis observed in patients with NSF. These observations will be strengthened if the phenotypic changes reported here in dermal fibroblasts cultured from patients with NSF can be reproduced by exposure of 'normal' dermal fibroblasts to gadolinium-containing contrast agents in vitro.

Del Galdo and colleagues observed differential expression of 551 genes when normal human monocyte-derived macrophages exposed to Omniscan were compared with macrophages exposed to phosphate buffered saline. ${ }^{22}$ They define a 'macrophage Gd signature' as those 31 upregulated genes with the highest signal intensity, of which three are interferon-inducible genes and five encode CC and CXC chemokines, most prominently CCL8/MCP-2. They extended these observations to NSF by demonstrating markedly increased CCL8/MCP-2 expression in affected skin from three patients with NSF compared with that in normal skin obtained from a healthy control individual. Common to the production of these chemokines is activation of nuclear factor $\kappa \mathrm{B}(\mathrm{NF} \kappa \mathrm{B})$. By inhibiting $\mathrm{NF} \kappa \mathrm{B}$

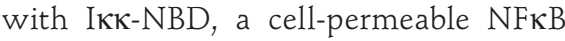
inhibitory peptide, they completely abrogated Omniscan-induced upregulation of CCL8, CXCL10 and CXCL11 and markedly reduced the upregulation of CCL12. These findings provide further evidence that a gadolinium-containing contrast agent can directly alter human monocyte-derived macrophage function in a way that could contribute to the development of NSF. Further investigation should be directed towards clarifying the mechanism by which gadolinium interacts with cells to trigger these changes in function, so that interventions might be developed to inhibit these processes and prevent the development of NSF following exposure to a gadolinium-containing contrast agent.

Since NSF was first observed over a decade ago, mounting evidence has associated the development of this devastatingly disabling condition with exposure of patients with underlying kidney disease to gadoliniumcontaining contrast agents used in imaging procedures. Thus, it is now time to rename this condition more precisely as 'gadolinium-associated systemic fibrosis' and to remove 'nephrogenic' from its name, since NSF neither originates in the kidney nor is caused by factors originating in the kidney. At present, no method is available to prevent gadolinium deposition in tissue; once it has deposited, gadolinium persists in tissue. ${ }^{17}$ Radiologists have developed guidelines to try to decrease the incidence of this condition, which include measuring the serum creatinine level before administering a gadolinium- containing contrast agent to an individual at risk for impaired renal function, avoiding the use of Omniscan or Magnevist in patients with GFR $<30 \mathrm{ml} /$ $\mathrm{min} / 1.73 \mathrm{~m}^{2}$ and injecting the lowest possible dose of a gadolinium-containing contrast agent when no alternative exists. ${ }^{23}$ However, the combined epidemiological and laboratory data convincingly support avoiding the use of these drugs in patients with renal impairment. Only by exercising tremendous restraint when deciding whether or not to administer a gadolinium-containing contrast agent to a patient with diminished renal function can this iatrogenic epidemic be brought to an end.

Provenance and peer review Commissioned; externally peer reviewed.

\section{Accepted 9 September 2010}

Ann Rheum Dis 2010:69:1895-1897

doi:10.1136/ard.2010.134791

\section{REFERENCES}

1. From the Centers for Disease Control and Prevention. Fibrosing skin condition among patients with renal disease - United States and Europe, 1997-2002. JAMA 2002;287:838.

2. Koreishi AF, Nazarian RM, Saenz AJ, et al. Nephrogenic systemic fibrosis: a pathologic study of autopsy cases. Arch Pathol Lab Med 2009;133:1943-8.

3. Kay J, Bazari H, Avery LL, et al. Case records of the Massachusetts General Hospital. Case 6-2008. A 46-year-old woman with renal failure and stiffness of the joints and skin. N Engl J Med 2008;358:827-38.

4. Todd DJ, Kagan A, Chibnik LB, et al. Cutaneous changes of nephrogenic systemic fibrosis: predictor of early mortality and association with gadolinium exposure. Arthritis Rheum 2007;56:3433-41.

5. Nazarian RM, Mandal RV, Kagan A, et al. Quantitative assessment of dermal cellularity in nephrogenic systemic fibrosis: a diagnostic aid. J Am Acad Dermatol. Published Online First:2010.10.1016/j.jaad.2010.05.034

6. Cowper SE, Su LD, Bhawan J, et al. Nephrogenic fibrosing dermopathy. Am J Dermatopathol 2001;23:383-93.

7. Jiménez SA, Artlett CM, Sandorfi N, et al. Dialysis-associated systemic fibrosis (nephrogenic fibrosing dermopathy): study of inflammatory cells and transforming growth factor beta1 expression in affected skin. Arthritis Rheum 2004:50:2660-6

8. James WD, Odom RB. Graft-v-host disease. Arch Dermatol 1983;119:683-9.

9. Grobner T. Gadolinium - a specific trigger for the development of nephrogenic fibrosing dermopathy and nephrogenic systemic fibrosis? Nephrol Dial Transplant 2006 Oct 11 [Epub ahead of print]

10. Prince MR, Arnoldus C, Frisoli JK. Nephrotoxicity of high-dose gadolinium compared with iodinated contrast. J Magn Reson Imaging 1996;6:162-6.

11. Perriss R, Løkkegaard $\mathrm{H}$, Løgager V, et al. Preliminary experience with contrast-enhanced MR angiography in patients with end-stage renal failure. Acad Radiol 2005;12:652-7.

12. Rydahl C, Thomsen HS, Marckmann P. High prevalence of nephrogenic systemic fibrosis in chronic renal failure patients exposed to 
gadodiamide, a gadolinium-containing magnetic resonance contrast agent. Invest Radiol

2008;43:141-4.

13. Abujudeh HH, Kaewlai R, Kagan A, et al. Nephrogenic systemic fibrosis after gadopentetate dimeglumine exposure: case series of 36 patients. Radiology 2009;253:81-9.

14. High WA, Ayers RA, Cowper SE. Gadolinium is quantifiable within the tissue of patients with nephrogenic systemic fibrosis. J Am Acad Dermatol 2007:56:710-12.

15. Pietsch H, Lengsfeld P, Steger-Hartmann $\mathrm{T}$, et al. Impact of renal impairment on long-term retention of gadolinium in the rodent skin following the administration of gadolinium-based contrast agents. Invest Radio/ 2009;44:226-33.
16. Hill AB. The environment and disease: association or causation? Proc $R$ Soc Med 1965;58:295-300.

17. Kay J, High WA. Imatinib mesylate treatment of nephrogenic systemic fibrosis. Arthritis Rheum 2008;58:2543-8.

18. Distler JH, Jüngel A, Huber LC, et al. Imatinib mesylate reduces production of extracellular matrix and prevents development of experimental dermal fibrosis. Arthritis Rheum 2007;56:311-22.

19. Akhmetshina A, Venalis P, Dees C, et al. Treatment with imatinib prevents fibrosis in different preclinical models of systemic sclerosis and induces regression of established fibrosis Arthritis Rheum 2009;60:219-24.

20. Wermuth PJ, Del Galdo F, Jiménez SA. Induction of the expression of profibrotic cytokines and growth factors in normal human peripheral blood monocytes by gadolinium contrast agents. Arthritis Rheum 2009;60:1508-18.

21. Piera-Velazquez S, Louneva N, Fertala J, et al. Persistent activation of dermal fibroblasts from patients with gadolinium-associated nephrogenic systemic fibrosis. Ann Rheum Dis 2010;69:2017-23.

22. Del Galdo F, Wermuth PJ, Addya S, et al. $N F \kappa B$ activation and stimulation of chemokine production in normal human macrophages by the gadolinium-based magnetic resonance contrast agent, Omniscan: possible role in the pathogenesis of nephrogenic systemic fibrosis. Ann Rheum Dis 2010;69:2024-33.

23. Thomsen HS, European Society of Urogenital Radiology (ESUR). ESUR guideline: gadoliniumbased contrast media and nephrogenic systemic fibrosis. Eur Radiol 2007;17:2692-6. 